\title{
Nobel Lecture: Defining and measuring optical frequencies*
}

\author{
John L. Hall \\ JILA, NIST, and University of Colorado, Boulder, Colorado 80309-0440, USA
}

(Published 17 November 2006)

\begin{abstract}
Four long-running currents in laser technology met and merged in 1999-2000. Two of these were the quest toward a stable repetitive sequence of ever-shorter optical pulses and, on the other hand, the quest for the most time-stable, unvarying optical frequency possible. The marriage of UltraFast and UltraStable lasers was brokered mainly by two international teams and became exciting when a special "designer" microstructure optical fiber was shown to be nonlinear enough to produce "white light" from the femtosecond laser pulses, such that the output spectrum embraced a full optical octave. Then, for the first time, one could realize an optical frequency interval equal to the comb's lowest frequency, and count out this interval as a multiple of the repetition rate of the femtosecond pulse laser. This "gear-box" connection between the radio frequency standard and any/all optical frequency standards came just as Sensitivity-Enhancing ideas were maturing. The four-way Union empowered an explosion of accurate frequency measurement results in the standards field and prepares the way for refined tests of some of our cherished physical principles, such as the time-stability of some of the basic numbers in physics (e.g., the "fine-structure" constant, the speed of light, certain atomic mass ratios etc.), and the equivalence of time-keeping by clocks based on different physics. The stable laser technology also allows time-synchronization between two independent femtosecond lasers so exact they can be made to appear as if the source were a single laser. By improving pump/probe experiments, one important application will be in bond-specific spatial scanning of biological samples. This next decade in optical physics should be a blast.
\end{abstract}

DOI: 10.1103/RevModPhys.78.1279

\section{OVERVIEW AND SUMMARY}

The view backward over some momentous developments often suggests a kind of certainty and inevitability that may not have been evident, even in the slightest form, when the story was going on. One modern trend is to focus on some particular research project-one which is so simple and transparent that the Manager can expect to be successful in the chosen research task. But such a project will likely have modest consequences: Surely its consequences were at least dimly visible from the beginning. By contrast, this "Optical Frequency Comb" capability has come "out of the blue" from a remarkable synthesis of independent "state-of-the-art" developments in four distinct fields: UltraStable Lasers, UltraFast Pulse Lasers, UltraNonLinear Materials and Responses, and UltraSensitive Laser Spectroscopy. These separate fields were alike in their shared-but independent-pursuit of advancing simple and effective technology for using electromagnetic signals for their own spectroscopic and other optical physics interests in the visible domain. After the Great Laser Technology Synthesis of 1999-2000, celebrated by the brief name of "Optical Frequency Comb," the Optical Toolbox has really blossomed. In respecting Alfred Nobel's interest and life's work, we may be more expansive and clear: the field of optics has blossomed explosively!

The resulting new capabilities are unbelievably rich in terms of the tools and capabilities that have been cre-

\footnotetext{
*The 2005 Nobel Prize for Physics was shared by Roy J. Glauber, John L. Hall, and Theodor W. Hänsch. This lecture is the text of Dr. Hall's address on the occasion of the award.
}

ated, and these in turn are reinforcing progress in these related contributing fields. For example, after the frenzy of the first generation frequency measurements, some of the Generation II comb applications now include lowjitter time synchronization between ultrafast laser sources, coherent stitching-together of the spectra of separate fs laser sources so as to spectrally broaden and temporally shorten the composite pulse, optical waveform synthesis for Coherent Control experiments, precision measurement of optical nonlinearities using the phase measurement sensitivity of rf techniques, coherently storing a few hundred sequential pulses and then extracting their combined energy to generate correspondingly more intense pulses at a lower repetition rate, etc. Attractive topics of research for Generation III applications include precise remote synchronization of accelerator cavity fields and the stable reference oscillators for Large Array Microwave Telescopes, and potential reduction of the relative phase-noise of the oscillator references used for deep space telescopes (NASA, VLBI, etc.). That's just part of the first five years.

So in the precision metrology field, what exactly could one say is different now? In the same way we have enjoyed for the last half-century powerful spectroscopy methods with radio-frequency signals (consider Magnetic Resonance Imaging as one of its useful forms), we now can use frequency-control methods for optical spectroscopy. But there is a really important difference: the number of cycles per second in the optical domain is roughly 10-million-fold greater than in the rf domain, even as the rf processes themselves are still a few million-fold faster than human perception scales. In essence, these large factors map into a corresponding improvement in resolution-our measurement capability. 
See the discussion below. With human senses we can perceive halves and quarters and tenths, and perhaps a little better. These capabilities are enhanced approximately by the product of these two large numbers, bringing us immediately into the garden of a few parts in $10^{14}$ metrology. We can do even better by averaging independent measurements.

\section{METROLOGICAL STANDARDS AND SCIENCE}

\section{A close and bidirectional connection}

On occasion, accumulation of progress in the details of some scientific enquiry leads us to a glorious new vision of some parts of our experience: basically a new insight or organizing principle becomes available. But behind this revelation normally is a huge amount of painstaking work, quantitatively stating experimental results, which normally are expressed in absolute units. Sometimes an experiment can provide its own internal calibration, but in the main we really need to have practical standards to reference the measurements against. Of course the Standards must themselves be reproduced and distributed before the scientific results can be confirmed by several labs. The best case is that the needed Standard is based on some fundamental physical effect, ideally a quantum effect, so it can be independently realized by different laboratories at the same accuracy. This standards-realization process is in a revolution itself (BIPM).

\section{The length standard and its relationship to frequency and} time

It is useful to discuss a bit about metrological Standards, which we can initially take to be the seven base quantities of the Système International d'Unités (International System of Units), or more briefly the SI, or "the Metric System." These are Mass, kg; Time, s; Length, m; Current, A; Temperature, K; Quantity of Matter, mol; Unit of Light Intensity (candela), cd. From these seven base units, another $\sim 30$ useful derived units can be defined. For our purposes of stretching measurement precision to the ultimate limits, clearly Time and Length are the two quantities offering the highest potential precision. For eons the day was a natural unit for Time, but standards for Length have seemed artificial and arbitrary. In 1791 the Metric System was first discussed but, lacking serious metrology experience, these Age of Enlightenment gentlemen of the French Academy of Sciences decided that the Metre would be defined as some small fraction $\left(\frac{1}{4} \times 10^{-7}\right)$ of the Earth's circumference on a great circle passing through the poles and France. Of course, having the standard based on surveying had some limitations in practical lab work, but at least the unit of length was finally a definite and basically absolute distance. This was a welcome change since public exhibits in places such as Braunschweig, Germany and on Crete Island, Greece show there was a succession of length standards in sequential use, as a new Duke of different personal arm length came into power. But by 1875, with the Treaty of the Metre Convention, a stable metal bar began to look like a good idea. While not fully universal and independently realizable, the factory could make many of these prototype Metre bars, and could confirm their equivalence.

The community of Metric countries in 1889 welcomed the improved X-cross-section meter bars known as the "International Prototype Metre" length standard. This design used graduations (lines) engraved onto a platinum-iridium bar, with a Meter defined as the separation between two graduation lines at $0{ }^{\circ} \mathrm{C}$, measured with a specified mounting arrangement, and under atmospheric pressure. The 30 new bars were calibrated using an optical comparator technique, before dissemination of two to each country.

By 1890 A. A. Michelson had identified the exceptional coherence of the $\mathrm{Cd}$ red line, and by 1892 had used it with his new interferometer to determine the length of the International Prototype Metre. His measurements showed that the defined Metre contained 1553164.13 units of the wavelength of the cadmium red line, measured in air at $760 \mathrm{~mm}$ of atmospheric pressure at $15{ }^{\circ} \mathrm{C}$. For this and other contributions, Michelson was awarded the Nobel Prize in 1907. Of course thermal expansion was a limiting problem, such that when the low-expansion steel alloy Invar was invented, the creator (and Director of the BIPM), C. D. Guillaume, was awarded the Nobel Prize for 1920. However, the SI Metre definition was unchanged for 85 years: the Meter Bars worked well and optical comparators got fatigueless photoelectric eyes.

Spectroscopic experiments and supporting Quantum Theory led to improved understanding and improved light sources. The metrological needs of the World Wars changed the Science climate, and transportation disruptions emphasized the advantage of having independently-reproducible standards based on quantum physics. Eventually, in 1960 the Eleventh General Conference on Weights and Measures was able to redefine the International Standard of Length as 1650763.73 vacuum wavelengths of orange light resulting from transitions between specified atomic energy levels of the krypton isotope of atomic weight of 86 . Going forward with a new definition, one would say the $\mathrm{Kr}$ wavelength is $\lambda=1 \mathrm{~m} / 1650763.73=0.605780211 \mu \mathrm{m}$. While the adopted Definition speaks about unperturbed atoms, in fact several shifts were observed in light from the discharge lamp used for realizing this Metre in practice. Pressure shifts and discharge operating conditions were stabilized by operating the lamp at a specified discharge current and at a fixed pressure and temperature (using the triple-point of liquid nitrogen). A field-induced gas flow of $\mathrm{Kr}^{+}$led to a wavelength difference of light viewed from the two cell ends. When laser comparisons with this standard were performed, the additional problem of radially-dependent Doppler shifts of the emitted light was discovered.

The 1960s and 1970s saw a number of different stabilized laser systems introduced, refined, and the wave- 
lengths measured and compared between various national labs. Basically, all these laser systems were entered into the competition to be the next International Length Standard. There were then 48 nations involved in the Metre Convention, so politically speaking choosing one out of the many offered candidate lasers would be difficult. In addition, none of these approaches were overwhelmingly superior, when performance, cost, and complexity were all considered. And scientifically, it seemed attractive for the new Length Standard definition to be based on the Speed of Light, introduced as a defined quantity. On the basis of a number of laserbased measurements, this value was taken as 299792458 m/s exactly, a rounded value in accord with the measurements of the several standards labs. This redefinition of 1983 took the following form: "the Meter is the length of the path traveled by light in vacuum during a time interval of 1/299,792,458 of a second. The speed of light is

$c=299792458 \mathrm{~m} / \mathrm{s}$, exactly.

The second is determined to an uncertainty, $U=1$ part in $10^{14}$ by the Cesium clock."

The General Conference also suggested several recommended radiations for realizing the meter at that time, e.g., "The wavelength of the iodine-stabilized Helium-Neon laser is

$$
\lambda_{\mathrm{HeNe}}=632.99139822 \mathrm{~nm},
$$

with an estimated relative standard uncertainty $(U)$ of $\pm 2.5 \times 10^{-11}$."

In all of these changes in definition, the goal was not only to improve the precision of the definition, but also to change its actual length as little as possible (see NIST). With the speed of light defined, an optical frequency (linked to time) can thus serve as a length unit.

\section{Fundamental physics issues in the redefinition of length}

At the times of these redefinitions, there were some concerns that we were switching the physical basis for the Metre definition. For example, if in the future we discover that some of the "constants of Physics" actually are slowly changing, one could worry that the new definition might impact or even limit our discovery process. In any case, we would be unaware of a global change that would conserve the physical relationships we have discovered. But could there be a differential effect that might be observable? Before 1960 we were accepting the spacing of some lattice planes in the Pt-Ir alloy of the Meter Bar as our measurement basis for length: this length certainly would fundamentally involve Quantum Mechanics, and Electricity and Magnetism. And, considering the thermal vibration of molecules in the somewhat-anharmonic interatomic potentials, we can suppose that the nuclear masses-and thus the Strong Interactions - will also play a role in length via the thermal expansion. With the 1960 redefinition of the Metre in terms of a Krypton atom's radiation wavelength, perhaps we were opening some opportunity for confusion?
Now Quantum Mechanics and Electricity and Magnetism are still fundamentally involved, but the atom's mass is involved only in a reduced-mass correction, rather than via thermal effects. Certainly a new "constant," the speed of light, is linearly serving as the dimensioned scale constant. Initially the 1983 redefinition appears to be still a different sort compared with the $1960 \mathrm{Kr}$ definition, but really it just repeats the energy level difference idea (now it is Cs in defining the second rather than $\mathrm{Kr}$ defining an optical energy) followed by a conversion of dimensions. Who knows if there is some fun hidden in here?

Where we have come to is that the SI is now functioning with six, rather than seven, basic units. The Metre has been demoted to a derived unit, and the significance of Time and Frequency have been further elevated. This begins a long story, with the SI base units being challenged by spectacular advances "at the bottom of a Dewar" (Flowers and Petley, 2001), giving us a Josephson-effect based voltage standard (Nobel Prize of 1973), while the von-Klitzing-effect defines a quantum resistance standard (Nobel Prize of 1985). Taken together as $V^{2} / R$, an electrical Watt unit is apparent, while an SI Watt-defined as a Joule per second-would be represented as $\frac{1}{2} \mathrm{~kg}(\mathrm{~m} / \mathrm{s})^{2} / \mathrm{s}$. The relationship between these is established by a "Watt Balance" experiment (Steiner et al., 2005). Recently the Single Electron Transistor begins to enable digital counting of electron charges per second, contacting the SI Ampere, the unit of electric current. This interface between metrology and quantum physics is becoming a "Hot Topic" of our time (BIPM; Borde, 2005). The remarkable advances in metrology also allow-and advances in Cosmology and Astronomy strongly motivate-curiosity about the "exactness" and "time-invariance" of the various physics numbers used in our description of physical reality.

\section{CLOCKS AND TIME}

Time represents our most precisely measurable quantity and so it always has attracted certain kinds of devoted researchers. But also, now with various sensors and microprocessor control, many physical parameters can be read out by frequency measurements, and so we add a huge number of scientists in other fields who want to recover the finest details within their measurements. (Still, many really important research subjects are not yet so well developed that these frequency tools are useful: for example, world-changing decisions about air pollution management are being made even though we scarcely are sure about the sign of some effects.)

But for technology people, the improvement of time measurement precision grows as a field of intense interest and competition worldwide. In no small part this is because of the very advances singled out by this year's Prize: a capability jump by several decades is uncommon in any field, let alone the field where the precision of measurement was already at the highest level, and had already been driven to near its apparently basic limits.

Of course interest in time has been part of man's his- 
tory from our beginnings, but only in the last several recent centuries have some lucky subsets of people been somewhat isolated from seasonal variations, with leisure to think about Nature, and so time as an experimental parameter began to emerge. Nowadays we can look from the scientific and experimental point at the question: why would one be interested in time? For those who love precision, the clear reason is that time is the most powerful metrological variable.

\section{Scaling of precision attainable when we are measuring time}

The precision of time measurements can be increased essentially without limit, by increasing the measurement duration and simply counting the increased number of cycles of some regularly spaced events. However, a stronger information growth with measurement duration is possible if we have a nice source that has coherence from the beginning of the measurement until the end. (For the present purpose we may take this "coherence" to mean that if we know the oscillation cycle's phase early in the measurement, the coherent source is so steady that the oscillation phase could be predicted at later times near the measurement's end to a precision of $1 \mathrm{rad}$ of phase.) In this case we can have a measurement precision which will grow with the measurement interval $\tau$ according to $\tau^{3 / 2}$. A simple way to explain this assertion is to suppose we divided the measurement duration into three equal sections, each with $N / 3$ measurements. In the starting zone we compare the reference clock and the unknown clock, with a relative phase imprecision which scales as $N^{(-3 / 2)}$. Next, in the middle section, we merely note the number of events, $N / 3$. In the last section we again estimate the analog phase relationship between test and reference waves, with a relative imprecision which is again $N^{(-3 / 2)}$. Subtracting the two analog phases increases the uncertainty of one measurement by a factor $2^{1 / 2}$ so, altogether, the relative precision increases as $(1 / 2)^{1 / 2}(N / 3)^{3 / 2}$. Thinking of a microwave frequency measurement, with a base frequency of $10^{10} \mathrm{~Hz}$, in a $1 \mathrm{~s}$ measurement we have a factor of $10^{5}$ potentially to win. Commercial counters already can register 12 digits in $1 \mathrm{~s}$ for a reasonable input signal. One can see there is just a huge gain in measurement precision if we can measure a coherent frequency source in a proper way: No wonder we have the situation where metrology scientists as well as philosophers, sailors, and farmers are interested in clocks and time and seasons (Sobel, 1995). Indeed our most powerful test of the existence of Einstein's predicted gravitational radiation comes from the observed shortening of the year of the Hulse-Taylor binary pulsar: orbital clock physics vs quantum frequency standard physics on the Earth. This marvelous work was celebrated by the Nobel Prize of 1993.

\section{What makes a clock?}

The three essentials of clocks are as follows: a source of regular events, a counter/integrator to totalize the events, and a suitable readout mechanism to present the current result to an interested human or machine. In many ways the frequency source is the most interesting part since it is intrinsically an analog system, where the design goal is to diminish as little as possible the intrinsic stability of some physical oscillation, in the course of reading out its information. In this game, nuance and subtlety count for a lot. It is customary that the performance of clocks based on some well-known source of regular "clicks" will be improved several orders of magnitude by the work of many people over many years, with the ultimate fate of becoming suddenly obsolete due to the introduction of a better kind of stable oscillator. The new idea must be a serious advance, since it must be competitive at the start of its life with the previous technology which has been enhanced and improved in many stages. Still, some technologies have had a long lifetime-for example, one can still buy a good wristwatch based on a torsional oscillator, even though this balance wheel concept was used by Ch. Huygens in 1675.

Keeping time has been of serious interest since man turned agrarian, but became of critical interest with the expansion of lucrative international trade: "inevitable" shipwrecks could be avoided by better knowledge of position (mainly longitude) at sea. Parliament's Longitude Prize of 1714 (above $\$ 10 \mathrm{M}$ in current terms) attracted John Harrison's attention and some 40 years of his inventive work. In 1761 his $\mathrm{H}-4$ clock demonstrated 1/5 $\mathrm{s} / \mathrm{d}, \delta \nu / \nu \sim 2.5 \times 10^{-6}$ even while at sea. This was severalfold better that the requirement, but only half the Prize was initially paid: in part the controversy was about the Intellectual Property. A second problem was conflict of interest within the judging Committee. [This story has been well told by Sobel (1995).] Present customers of precise timekeeping include TV Networks (for synchronization), cellular telephone companies, the GPS users who need the limiting performance, radio astronomers, NASA Deep Space Tracking, and various other branches of Science in which a physical variable has been read out by frequency methods.

\section{Evolution of frequency sources: Distinguishing precision and accuracy}

In discussing the performance of a mechanical clock, or the electronic oscillators based on vibrational modes of quartz crystals, it is clear that the basic frequency is set by mechanical dimensions. Such a device could be stable and have good precision, in that its readout could be determined with many digits, but there can be no claim to any particular fixed or natural frequency. Still the stability of any particular crystal device could be remarkable: a drift of $>10^{-6} /$ day gradually improved to the present $\lesssim 1 \times 10^{-10} /$ day, while the shift with acceleration remains near $10^{-9}$ per "g." The high frequency of electronic oscillators served well for convenient interpolation between clicks of the absolute standard, provided by zenith sightings of the daily motion of the Sun, as codified by the 1875 Metre Convention. (Later the Earth 
rotation data series were based on telescopic observations of the lunar occultation starts of various stars and planets.) By the 1950s the electronic oscillators were refined enough that variability $\sim 10^{-8}$ was inferred in the Earth's spin rate, and was associated with changes of the Earth-atmosphere system's moment of inertia due to North-South ocean tides, and large storms. The community wished to eliminate the variability, but still needed an absolute and universal (rather than local artifact) standard. The new choice in 1960 adopted a stated number of seconds in the "Tropical Astronomical Year 1900." Perhaps this was good in its motivation, in that the rotation of the Earth around the Sun would have a lower level of perturbation. However, a clock/oscillator that has only a single click per year will be hard to enjoy at its full precision. As a metrology principle we rather would prefer the basic frequency source to be at a very high frequency so that the integer multiple of the standard's clicks will be a huge digital number in our measurement of some interesting phenomenon, and the unavoidable noise and uncertainty of the remaining analog subdivision of the unit will be as insignificant as possible.

\section{Electronic clocks based on quantum transitions}

Based on Otto Stern's atomic beam method, which had resulted in his Nobel Prize of 1943, I. I. Rabi introduced atomic beam resonance methods which allowed probing internal (hyperfine) quantum energy states of atoms such as Cesium with greater precision. This work was recognized by the Nobel Prize of 1944 . Using atoms in this way, the independent realizability and universality requirements for a Primary Standard could be well addressed. In addition, the transition frequencies were near the high-frequency-end of the usable rf spectrum, so the Metrology aspects were optimized as well. The first Atomic Beam Clock was developed at NBS in 1949 based on microwave transitions in Ammonia, and by 1955 Cs beam clocks were in operation at the NPL and NBS. The powerful Method of Separated Oscillating Fields was invented by N. F. Ramsey, reported in 1955, and later recognized by the Nobel Prize in 1989. In this dual-excitation concept, suitable atoms were excited once, and then left to evolve their internal phase (ideally) free of perturbation, until a second excitation pulse effectively completed the interferometric comparison of the phase evolution rates between the atomic and laboratory oscillating systems. Progress on the Cs beam atomic frequency standard was widespread and rapid, allowing redefinition in 1967 of the SI Second as 9192631770 units of the Cs hyperfine oscillation period. Correspondingly, the Cs oscillation frequency is defined as (exactly) $9192631770 \mathrm{~Hz}$ (cycles of oscillation per second). The specialists involved in this redefinition of Time and Frequency wisely did not specify exact details of the measurement process, leaving room for considerable progress. For example, when laser-based optical pumping of atoms between hyperfine states became feasible and popular in the early 1990s, NIST colleagues built a new atomic beam cesium standard, NIST-7, based on optically transferring most of the population from the 16 available hyperfine levels into the special $(|3,0\rangle)$ lower state involved in the clock transition. Along with this factor, $\sim 16 \times$, improvements of the atom source itself, and better frequency source and readout electronics were helpful. Above all, computer-based signal processing and active control of measurement systematic offsets made it possible to reduce the inaccuracy of realizing the Cs second at NIST to $\sim 5 \times 10^{-15}$. But as usual in the artform of Precision Measurement, this "tour de force" system was soon made obsolete in a single step by a qualitatively better technology.

As shown by Kasevich, Riis, and Chu (1989), laser cooling of the Cs atoms made it possible to successfully implement the "atomic fountain" concept for the realization of the Cs-based frequency definition. By shifting laser frequencies or powers, a slowly moving ball of atoms could be dispatched vertically upward through the excitation rf cavity, reaching apogee a good part of a meter above the cavity, and then beginning the return trip to pass through the excitation cavity a few $100 \mathrm{~ms}$ later. With such a long coherent interaction time, instantly the resonance linewidth dropped to $\sim 1 \mathrm{~Hz}$, down from $\sim 300 \mathrm{~Hz}$ in the previous epoch of thermal beam of atoms. Optical probing of the atoms below (and temporally after) the cavity could yield the excitationprobability vs probe-frequency-tuning curve needed to control the source oscillator's frequency. By using suitably closed optical transitions for readout, one can have many photons emitted per atom so that, even after solid angle and detection inefficiencies are considered, the measurement noise is not much larger than the minimum associated with the finite number of atoms. Andre Clairon and his colleagues made the first real Cs Fountain Frequency Standard, in 1995 (Clairon et al., 1995) at the Paris Institute now known as LNE-SYRTE (Laboratoire National de Métrologie et d'Essais-Systèmes de Références Temps/Espace). Even without the contemporary schemes to break this atom-shot-noise limit, the fountain Cs clocks at NIST and SYRTE now achieve accuracy levels below $1 \times 10^{-15}$ when all the known measurement and perturbation issues are taken into account (Heavner et al., 2005). Of course with the resolution improvement one hopes for more potential accuracy, but will have beforehand an expanded list of small shifts and niggling concerns to consider. After all, even with the extended interaction time, fewer than $10^{10}$ oscillation cycles are counted, so the achieved inaccuracy of 1 $\times 10^{-15}$ already corresponds to $10 \mathrm{ppm}$ splitting of the atomic fountain's resonance linewidth. Fountain Cs clocks are limited by two newly important effects, collisionally induced frequency shifts due to the hugely increased atom density (Gibble and Chu, 1993), and shifts due to the effects of the ambient thermal radiation associated with the vacuum system's walls. Attempting to 
split lines further always brings a diverging list of new small problems, leading to an effective barrier.

An important observation is that for many types of Quantum Absorber samples the line broadening processes will be the same for both radio and optical frequency domains. For example, the atomic fountain apparatus could explore optical transitions, rather than microwave ones, with the same interaction time. Clearly we would prefer the higher base frequency of the optical world, since the resonance feature of interest will then display a relative sharpness increased by roughly the same huge factor of optical/microwave frequency ratio. With sharper line shapes we can expect more precise measurements that will let us better see the small effects of various experimental parameters, leading to better independent reproducibility which, with major investment of efforts, can often be parlayed into nearly a corresponding increase in measurement accuracy capability as we come more fully to characterize the offset processes. But before the Millennium Year of the Optical Comb, just how did you plan to measure the absolute optical frequency?

This repeatability idea seems weaker than the gold standard of accuracy, which additionally conveys our being able to connect the measured result with the base units of the Système International. But in fact we now know several optical clock systems that have tenfold smaller uncertainty than the Cs standard. So before a redefinition is appropriate, their comparisons will be most interesting, especially as an entry point for one of the most interesting branches of Science, trying to figure out which physical "laws" are essentially exact, which ones are ignoring some details to have a tidy presentation, and which are in fact stating "facts" about Nature which are not exactly actually true. Celestial mechanics, ideal gas laws ignoring molecular volumes, and parity conservation in atomic physics could be my examples.

\section{STARTING THE DREAM OF OPTICALLY BASED CLOCKS}

\section{The laser arrives}

The future of metrology was changed fundamentally on 12 December 1960 when a small team at Bell Labs, led by Ali Javan, eventually found the right conditions for their Optical Maser to generate self-sustained Optical oscillations. Their specially crafted gas discharge tube had the improbable situation in which the populations in two particular Neon atomic levels were reversed from the thermal norm: by means of the discharge in the more-abundant He gas, collisional energy transfer set up a population inversion, whereby more atoms were in the Ne's higher energy state. It is impressive that these conditions were established on the basis of careful measurements and modeling of the discharge conditions. Having the populations inverted from the usual case reverses the sign of the absorption that experience teaches us is a universal property of (normal) matter. Accordingly, with an inverted population, rather than absorption, Javan's group had optical emission. The atoms would provide amplification of any resonant optical signal passing down the discharge cell. A few percent gain would not be very exciting normally, except that the utilized multilayer mirrors were designed and fabricated to have reflection losses that could be even smaller, setting the stage for a buildup of power on every pass. So finally they did obtain a self-sustained continuous optical oscillation, and observed the collimated beam that was anticipated by Charles Townes and Arthur Schawlow in a classic paper of 1958. Similar ideas were also considered in the former Soviet Union, leading to the Nobel Prize of 1964 being shared by N. Basov, A. Prokhorov, and C. Townes.

\section{Connection to Glauber's coherent states of light}

In planning a theoretical study of optical fields, perhaps one can understand starting with known results for single-photon fields, then adding a few photons cautiously to see what happens. Actually, for all of us following Professor Glauber's work it was surprising just how few photons were needed for the new photon density distribution functions to change fundamentally from the customary Poisson limit: with increasing number of photons in a mode the fields start showing the small fractional fluctuations that would characterize a classical field. On the experimental side, for Javan's very first laser, the output laser power was $\sim 1 \mathrm{~mW}$, about $10^{16}$ photons per second. We can proceed to estimate the expected fractional variation of $1 / N^{1 / 2}$, but with such an incredibly large number of coherent photons in one mode, the result is an unphysically small variation. Thousands of merely technical processes would cause fluctuations larger than the predicted $1: 10^{8}$. An equivalent statement is that these lasers were operating strongly, far into the domain of classical fields, and quantum fluctuations would be very hard to observe. Indeed it was not until the end of the 1970s that people began again to appreciate how to study manifestly quantum fields with just a few photons in them. At this vastlyreduced intensity, quantum correlations are challenging to observe, but they are very interesting, since they correspond to rather significant fractional effects. For example, H. J. Kimble's group used phase-dependent Squeezed Light to make a spectroscopic measurement with about twofold better Signal/Noise $(\mathrm{S} / \mathrm{N})$ than the naive shot-noise limit (Polzik et al., 1992). To observe strong Squeezed Light effects, it is essential to minimize optical losses, as they work to revert the statistics toward the thermal limit. Regrettably, noise from technical sources will grow linearly in the laser power, while the advantage due to squeezing will grow more slowly. It seems that getting a factor of 10 amplitude S/N improvement will be incredibly difficult.

\section{Coherence of the laser field enables frequency diagnostics}

The Bell Labs laser design success had grown out a semiclassical view of how Optical Masers would operate. 
Yes, amplification would be provided by quantum mechanical atomic systems, rather than radio tubes or klystrons, and yes, each atom could contribute just one photon to the field in each event. But still, considering how huge is the number of photons in the field, the discreteness probably will hardly matter. Almost immediately the Bell-Labs team was testing this understanding by combining two separate laser beams into a single coaxial beam, and shining this onto the sensitive surface of a high-speed photodetector. They already were thinking of each laser oscillation as being an essentially classical field, satisfying reflection boundary conditions at the two mirrors. So this stable-and-repeating bouncing specification would define the possible wavelength(s) of the generated laser light. By luck and design the discharge was wonderfully calm, so one could expect the gas's refractive index would be essentially constant. Thus the interferometric boundary conditions would essentially define the oscillation frequency and, accordingly, one would expect to see a sharp optical frequency come out of this device. With two lasers' sharp frequencies on the nonlinear detector's surface, one should expect the difference frequency to be generated, which it was. I can still remember hearing the audio beat whistle that Javan had recorded when his two lasers were tuned almost to the same optical frequency. It was a $\sim 1 \mathrm{kHz}$ difference between two sources at $260 \mathrm{THz}$.

Actually the linewidth of these beats was remarkably narrow. We already expected that based on the numbers noted above a stream of $\sim 10^{16}$ photons/s would have random power fluctuation of $\sim 10^{-8}$ relative to the full power. So the optical phase could be extremely well defined. However, the laser's Schawlow-Townes linewidth calculation includes the role of optical loss, which actually limits the laser coherence, giving $\sim \mathrm{mHz}$ linewidth expectation.

In principle then we have a radiation of incredible sharpness, and should be ready to seek interesting physical effects. The immediate disappointing truth is that this tiny predicted laser phase fluctuation will be completely masked by noise of technical origins. We already noted that the strongest definition of the oscillation's frequency is fixed by the interferometric standing-wave condition bouncing on the laser cavity mirrors. But the lab is a noisy place, seismically speaking, with a quiet lab having a ground noise of $\sim 3 \times 10^{-9} \mathrm{~m} / \sqrt{\mathrm{Hz}}$ in the vibration frequency band, say, $1-30 \mathrm{~Hz}$. A laser cavity is some fraction of a meter in length, so it will be difficult to make a system arbitrarily stiff. Rather, some important fraction of the ground noise will appear as cavity length variations, and therefore laser frequency variations. Suppose we say only $1 \%$ couples in to relative length changes. One can instantly see the scale of the problem: $\sim 10^{-10}$ fractional frequency variations will be our a priori scale. Even temperature variations will be painful, since the $10^{-10}$ scale already corresponds to a few $\mathrm{mK}$ temperature change for low expansion materials like fused silica. We can make progress by locking the laser to a stable reference cavity (Drever et al., 1983). Optimizing for vibrational integrity, we will use a stiff structure for mounting the reference cavity mirrors, and then mount the assembly with a horizontally soft suspension. By focusing on the vibration isolation, Bergquist has obtained (Young et al., 1999, 2005) a record narrow laser linewidth $\sim 0.16 \mathrm{~Hz}$. Another approach seeks to minimize the cavity acceleration sensitivity. By use of a vertically-symmetric mounting (Notcutt et al., 2005) of the reference cavities, our group recently reported Hz-level laser linewidths.

\section{Coherence of the laser beats enables frequency-based laser control}

Considering the small intrinsic phase noise of the laser source, and the rather high power $\sim \mathrm{mW}$, heterodyne detection of the beat frequency between two laser sources yields an interestingly high Signal/Noise ratio. Even with very short averaging times, say $1 \mu \mathrm{s}$, we have generous $\mathrm{S} / \mathrm{N}$ performance. Additionally, for such short times a well-engineered laser will scarcely respond to the "garbage effects" of real life in the lab (temperature variations, power-supply variations, vibrations, etc.) within $1 \mu$ s these have not changed the system very much. The duration of the perturbations is too small for them to begin to wreak havoc with the stability of the frequency-defining cavity. So we actually can make useful measurements of the laser's phase in such a quick time frame that the problems are not yet apparent. One begins to see a strategy coming up: We will quickly measure what the laser actually is doing, compared with our desired behavior, and then use feedback onto suitable actuators to control the laser's frequency. If we can make the corrections quickly enough and accurately enough, then the controlled laser will very closely approximate the ideal frequency-stable laser we need.

Implementation of this servo-control feedback concept is a multi-nuanced thing, in the perfection of which this author has invested something over 40 years of active work. It has led to a lot of interesting and useful electrooptic tools and techniques.

\section{The relative high power of lasers empowers nonlinear spectroscopy and sharp resonances}

Let's begin with the first approach to observation of narrow atomic resonances, using Saturated Absorption Spectroscopy. These phenomena were studied first within a laser cavity by Bill Bennett using the dispersion effects associated with the active neon laser gas. Owing to the Doppler effect, the Neon atom's natural resonance linewidth of $\sim 10 \mathrm{MHz}$ becomes masked and broadened to $\sim 1500 \mathrm{MHz}$. Thus most of the gas atoms are detuned, and in a velocity-specific way. Some atoms have velocities near the special one giving the Doppler shift that will bring them into resonance with the intracavity laser field. Actually there are two such velocities to consider, since the laser beam goes both directions as it is bouncing back and forth between the mirrors. These resonant atoms will interact rather strongly with the field, leading to an increased decay rate for excited state 
atoms of that velocity-their inverted population gets converted into cavity photons. If we imagine a plot of the population difference (upper state minus lower state populations) we can expect to see a local and rather narrow dip around the velocity which is being converted from population inversion into light quanta. Actually there are the two mirror-symmetric dips as noted before. The interesting effects come when we let the laser frequency be tuned toward the atom's rest-frame frequency. Then the resonant atoms will have lower and lower Doppler velocities, until finally the selected velocity is zero. Now a new thing happens: when detuned, we had two groups of active atoms contributing their power to the laser output. When we reach the central tuning, both running-wave fields interact with a single atom velocity group. So with fewer atoms contributing, the laser power decreases conspicuously, but only at the central tuning. This feature in the power output with laser tuning could be used for locking the laser to this central tuning dip, which is called "Lamb's dip" after Willis Lamb whose early theoretical work made clear this origin of the experimentally-observed effect. (His Nobel Prize in 1955 was for his work on the new sub-hyperfine structure in the Hydrogen spectrum.) As it turns out, operating pressures for optimum laser operation were rather large ( $\sim 3$ Torr, $400 \mathrm{~Pa})$, which led to substantial probability of atom-atom collisions, even during the few tens of ns optical lifetimes. So the Lamb dips would be broader and less deep, and had to be observed against a somewhat-peaked Doppler profile representing the distribution of available atom velocities. In addition to reducing the Lamb-dip contrast, significant frequency shifts were generated (Hall, 1968). One could not arbitrarily reduce the gas pressure since the discharge pumping mechanism actually populated a metastable $\mathrm{He}^{*}$ level, and collisions were needed to transfer this excitation to the Neon atom component in the discharge. So even though the wavelength of the laser's characteristic coherent light was more-readily-measurable than the incoherent light from the krypton discharge lamp (the existing wavelength standard), in fact the lasers' pressure shifts were simply too large to accept. Particularly this was the case since the discharge technology of the day led to important change of the fill gas pressure and species ratio with operation, due to electrode sputterpumping.

The clearly important idea of separating the amplifier and the reference gas cells' functions was soon introduced by Lee and Skolnick. More discussion of those interesting developments is available elsewhere (Barger and Hall, 1969; Hall, 2000), but for our present purposes we do need to consider some of the essentials. Since the purpose was to have a sheltered life for our reference atoms, it was attractive to be thinking in terms of $a b$ sorption, rather than amplification. Then we did not need any discharge or optical pumping of the reference quantum resonators. Of course, to be able to use Lamb's nonlinear resonance for frequency stabilization, we certainly needed to be able to tune the laser to the resonance frequency of the reference gas. Nowadays, this is no big problem, by just using tunable lasers. At that time, the best idea to get a wavelength coincidence would be to use molecules as the absorbers - then we would have zillions of absorption lines to choose from. The modern champion for this approach is molecular Iodine, with narrow useful absorption lines from the near infrared (IR) down to $\sim 500 \mathrm{~nm}$. For other molecules, utilizing transitions only between vibrationalrotational states, typical wavelengths are in the IR from $\sim 2$ to $10 \mu \mathrm{m}$ range.

The first such dual-component optical frequency reference system, and still one of the better ones, uses a $\mathrm{HeNe}$ discharge cell to provide gain and laser oscillation at $3392 \mathrm{~nm}$ (Barger and Hall, 1969). Also contained in the laser cavity is a cell containing $\mathrm{CH}_{4}$ molecules, plain old tetrahedrally-symmetric methane, which has interesting lines that can be reached with the $\mathrm{HeNe}$ laser. To be brief, the necessary emitter/absorber spectral overlap is arranged by selection, based on good luck. The IR absorption band utilized, $\nu_{3}$, is a strong fundamental vibration band, providing $0.18 \mathrm{~cm}^{-1}$ absorption coefficient per Torr. Of course having the absorber gas inside the cavity means we do not need very much absorption to have an impact on the laser dynamics-just a few percent would be fine, since it would then be roughly $\frac{1}{2}$ the loss associated with the output-coupling mirror. At 10 mTorr, the associated pressure broadening of the $\mathrm{CH}_{4}$ resonance would then be $\sim 160 \mathrm{kHz}$, similar to the $130 \mathrm{kHz}$ broadening associated with the molecular freeflight through the intracavity light beam, of $0.3 \mathrm{~mm}$ typical diameter. Importantly, the pressure-induced shift turns out to be very small for these transitions, only $\sim 1 \mathrm{kHz}$ under these conditions.

So we are talking about a system with a resonance in the power curve of $\sim 0.6 \mathrm{MHz}$ FWHM, with perhaps $5 \%$ relative contrast on the total laser output of say $200 \mu \mathrm{W}$. A little calculation leads one to a Signal/Shot-Noise ratio $\sim 10^{6}$ in a $1 \mathrm{~Hz}$ measuring $\mathrm{BW}$, while we are looking at the sub-MHz-wide peak produced at the central tuning, when both cavity running waves are bleaching the same absorbing molecules and thereby reducing the intracavity absorption losses. If this $\mathrm{S} / \mathrm{N}$ were optimally used, the laser could be stabilized to have sub-Hz frequency deviations measured in $1 \mathrm{~s}$ intervals. In 1968 when this Saturated Absorption Optical Frequency Reference business began, our detectors and preamplifiers were not so good, and we did not begin to approach the shot-noise limit - that would have been a frequency (in)stability of $\sim 2 \times 10^{-14}$ at $1 \mathrm{~s}$. Early on, we did get $\delta \nu / \nu \sim 1 \times 10^{-12}$, which was soon improved to $3 \times 10^{-13}$ with better detectors and signal processing.

By locating the sample cell outside the laser resonator, the physical situation could be more-readily analyzed, and this arrangement was employed by Bordé, Hänsch, and Chebotayev's group in early experiments. The interesting details are discussed in textbooks; see, e.g., Letokhov and Chebotayev (1977), Stenholm (1984), and Levenson and Kano (1988). Now we consider the transittime linewidth issue. 
Free-flying molecules see a light pulse: Two views of the Uncertainty Principle

For these transitions, the radiative lifetime $(\sim \mathrm{ms})$ was much larger than the transit time of the essentially freeflying molecules in crossing the laser beam. At low pressure the saturated absorption linewidth was not collisionally nor Doppler limited, so it could be immediately observed that the resonance linewidths could be reduced by increasing the field/molecule interaction time. Larger beams helped. So did liquid Nitrogen cooling of the glass cell. So a serious study began to really understand the line shape in the free-flight regime. Chebotayev and his colleagues developed the theory analytically near the low-pressure, low optical power limit (Bagaev et al., 1976). The JILA theory was based on computer integrations of the Density Matrix for absorbers making a free transit through the assumed Gaussian light beam mode (Borde et al., 1976). Low intensity and weak interactions were assumed to simplify the calculations, but soon it became clear that most of the observed signal would be contributed by a very small number of slow molecules. The theoretical result is a logarithmic cusp at the exact line center. With long interaction times, even a "weak" power would lead to saturation and other strong-field effects.

We need low velocity in the longitudinal direction so that the molecule would not cross wave fronts axially, and thereby begin to develop Doppler-related phase modulation. Effectively molecules should fly perpendicular to the axis, and leave the wave front after the transit with only $<1$ rad geometrical phase shift. We also need low transverse velocities, since a longer transit time will be directly imaged into a narrower line. We can see $\delta \nu \cdot \tau \approx 1$ will yield $\delta \nu=\beta \mathrm{v}_{\text {th }} / \mathrm{w}_{0}, \delta \nu$ is the HWHM of the observable resonance, $\mathrm{v}_{\text {th }}$ is the thermal velocity, $\mathrm{w}_{0}$ is the Gaussian beam radius, and $\beta$ is a measured parameter. Experimentally we found $\beta \mathrm{v}_{\text {th }}=88 \mathrm{kHz} \mathrm{mm}$ for Methane at room temperature. Laser mode radius $\mathrm{w}_{0}$ values from $56 \mu \mathrm{m}$ to $9 \mathrm{~cm}$ were measured, with corresponding HWHM values from 1.6 MHz down to $940 \mathrm{~Hz}$ (see Fig. 1). (The interesting substructure will be addressed momentarily.) First it is useful to consider the transit-time broadening in the Fourier-dual domain: angular divergence. Corresponding to a Gaussian beam radius $\mathrm{w}_{0}$ there is a minimum angular divergence of the collimated laser beam of $\delta \theta=\lambda / 2 \pi \mathrm{w}_{0}$. The $k$-vector spread particularly the nonaxial components lead to a velocity-dependent Doppler shift of the same sign for both running waves, which will appear as broadening and shift of the resonance. Of course with a smaller mode diameter, the angular content is increased, and more broadening will appear spectrally.

While molecules typically do not have the "closed" optical transitions analogous to those needed for normal laser atom cooling, polar molecules do have a dipole moment. So with some electrical effort, one can arrange Sisyphus-like molecule slowing by switching the sign of the strong applied electric field, as shown by Meijer's group (Bethlem et al., 1999). More recently Ye's group

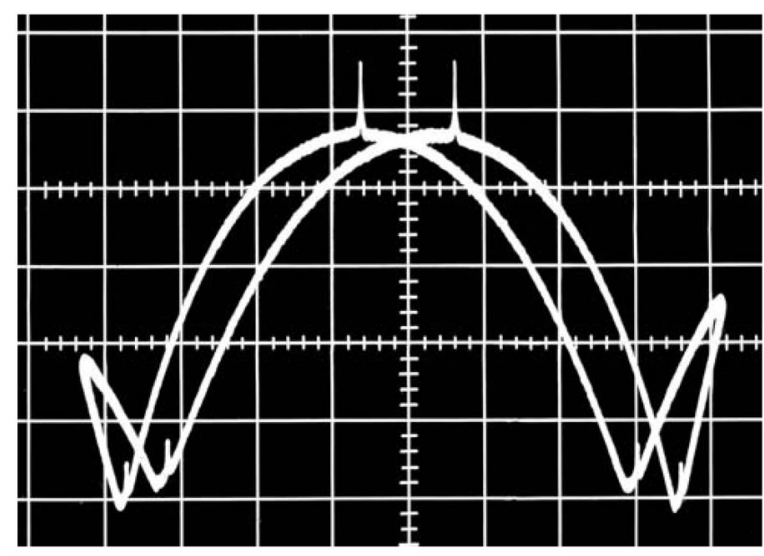

FIG. 1. Saturated absorption peak in $\mathrm{CH}_{4}$ molecules. HeNe laser at $3.39 \mu \mathrm{m}$ is excited by rf discharge. $\mathrm{CH}_{4}$ cell at 12 mTorr (16 mbar) is located inside laser cavity. Power output is $300 \sim \mu \mathrm{W}$ and peak contrast is $\sim 12 \%$. Peak width is $\sim 270 \mathrm{kHz}$ HWHM. At maximum power $(\sim 0.8 \mathrm{~mW})$ contrast is $\sim 15 \%$. Cavity free spectral range is $250 \mathrm{MHz}$. Note crossover resonances in two-mode region near cusps. Hysteresis of scan causes trace doubling.

has achieved unprecedented high resolution microwave spectroscopy on Stark-slowed $\mathrm{OH}$ free radicals (Hudson, 2006). Certainly this will be an interesting frontier.

Other important directions are high sensitivity detection and improving the accuracy of locking to the molecular signals. For example, some JILA work (NICEOHMS) shows a road to sensitivity increase by combining cavity enhancement and rf sideband techniques (Ma et al., 1999). A fascinating physics avenue is the search for a parity-related frequency shift between suitable enantiomers (Ziskind et al., 2002). Other important laser applications are considered in Svanberg's book (Svanberg, 1992).

Momentum transfer from light to molecules-The recoil splitting

A full treatment of radiative interactions must include the field and molecular momenta, as well as the photon numbers and internal states of the quantum system. Such a treatment is essential for the case of pumping atoms with closed energy levels, which can allow the repeated interactions and deep velocity cooling celebrated by the 1997 Atom Cooling Prize of Phillips, Chu, and Cohen-Tannoudji. For the molecular sample of interest here, there are many decay channels, and likely even impact on the vacuum chamber walls before any particular molecule reappears in the laser fields: so a single interaction picture is reasonable. A clear observation of the transfer of momentum from field to atomic system is available with Saturated Absorption Spectroscopy, basically because it is a two-step process. Let's consider absorbers that initially have essentially zero velocity along the light beam. Then the left-running light beam can be tuned to $v=\nu_{0}\left(1+h \nu / 2 M c^{2}\right)$, the extra (recoil) energy being needed beyond the transition energy $\nu_{0}$ to provide the kinetic energy associated with the re- 


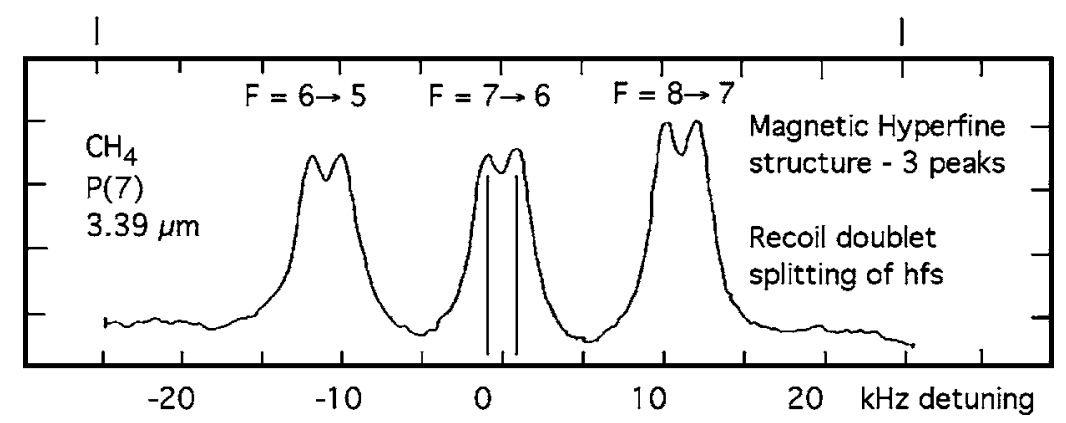

FIG. 2. Recoil Splittings of HyperfineStructure Peaks in free-flight Methane Molecules. The vertical strokes indicate the positions of the two recoil components in one of the Hyperfine components.

coil momentum the molecule will have after the transition occurs. The opposite-running beam will also deplete this zero-velocity group. So at this resonance tuning the resulting nonlinear decrease of molecular opacity will lead to a peak in the transmission spectrum, and it is shifted slightly to the blue of the rest frequency. Another interesting case occurs when the molecules have a velocity $\mathrm{v}=h / M \lambda$, i.e., there is enough molecular momentum initially so that when the red-detuned laser interacts with this molecule, the photon and molecular momenta just cancel, and the original kinetic energy can make up for the photon's energy deficit. The result is an excited molecule with zero axial velocity. Now the laser beam in the other running direction will experience amplification from this particular tuning condition, again leading to a relative peak in the sample's transmission. With the molecule initially possessing some kinetic energy, the laser tuning for this upper-state resonant condition will be $\nu$ $=\nu_{0}\left(1-h \nu / 2 M c^{2}\right)$. So considering photon recoil, the nonlinear interaction is associated with either the ground or excited state population being accessed by both beams for the same detuning, namely, zero velocity in either one of the two states. For methane the splitting between the two peaks is $2.163 \mathrm{kHz}$ and may be seen clearly in Fig. 2 (Hall et al., 1976).

While the JILA and University of Paris Nord work exploited mainly the large diameter optical beams to gain a longer molecular interaction time, Chebotayev, Bagayev, and colleagues in the Novosibirsk group made good use also of another physical idea, namely, the use of super-slow molecules to contribute the main part of the observed signal. In this way an additional 20-fold linewidth reduction to $<50 \mathrm{~Hz}$ was achieved (Bagaev et al., 1991). An important aspect of this approach is that the total 3D effective molecular temperature is below $0.1 \mathrm{~K}$, leading to a much-reduced second-order Doppler shift of $\ll 1 \mathrm{~Hz}$. An average velocity $13 \times$ below thermal for slow $\mathrm{C}_{2} \mathrm{HD}$ molecules was shown by Ma et al. (1999) and was feasible only because of the very large sensitivity provided by the NICE-OHMS technique.

\section{Other optical frequency references based on nonlinear spectroscopy}

Many research groups have been attracted to working with laser stabilization for Measurement Standard applications, such as interferometric calibration of gage blocks that serve to check reference standards used by industry. For this kind of application it is highly desirable that the reference laser beam be visible, as well as stable enough and reproducible enough. A huge success in this area is the $633 \mathrm{~nm}$ HeNe laser with an intracavity Iodine cell, and well developed systems of this type are even available commercially. This $\mathrm{HeNe} / \mathrm{I}_{2}$ system was the one whose frequency was measured by the NBS efforts in the early $1980 \mathrm{~s}$, with an uncertainty of $70 \mathrm{kHz}$. (Being the first measurement of such a visible system, it is perhaps understandable that several of the uncertainties were far from fundamental in their origins.) Other labs joined in and over the next decade many labs gained experience and a few had frequency measurements confirming the NBS result. Slowly it became acceptable to reconsider the definition of the International Unit of Length, the SI Metre.

As may be seen, the world of spectroscopy offers us an unending garden of fascinating details. Presumably Parity-Non-Conservation will lead to a next generation of fine structures in chiral molecules, particularly with the development of cold-molecule techniques. But enough about the "ticks" of the clock: now we must return to the main story, the development of frequency stabilization and cycle-counting measurement toolsThe inside Gear-Works of the Optical Clock.

\section{MEASURING OPTICAL FREQUENCIES WITH OPTICAL COMBS}

The Metre redefinition of 1983 was not really a kindness to metrologists tasked with actually measuring some physical parts, because the practical methods for application to measurements were not yet spelled out. But it was a boon to the metrology researchers: it became their task to explore just which good stabilized laser system would have the optimal properties for precision interferometry, for outdoor surveying, for servoloop guidance of milling machines, etc. So within a dozen years after the redefinition there were at least ten well-developed optical frequency standards, as illustrated in Fig. 3.

As may be seen in Fig. 3, there are stable frequency sources available from roughly $10 \mu \mathrm{m} \quad(30 \mathrm{THz})$ to $\sim 280 \mathrm{~nm}(\sim 1 \mathrm{PHz})$, well beyond the visible range. It was striking that the difference between lines were surprisingly similar frequency intervals, $\sim 88 \mathrm{THz}$, approximately the frequency of the $\mathrm{CH}_{4}$-stabilized laser. This led to schemes where doubled frequency of one laser 


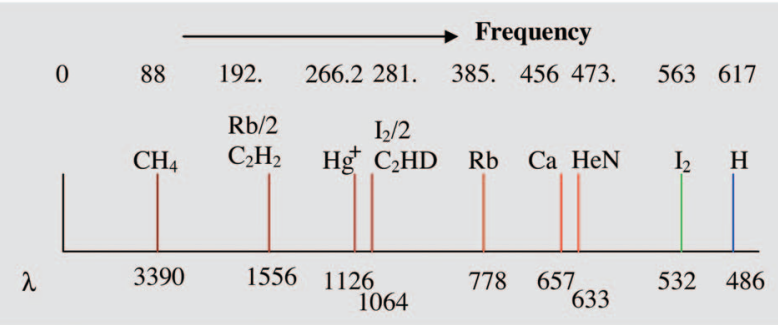

FIG. 3. (Color) Stable Lasers based on NonLinear Doppler-free Resonances in Gases (1995). The frequency axis (above) is in $\mathrm{THz}$ units, the wavelength scale (below) is in $\mathrm{nm}$.

would be compared with the sum of the two straddling lasers. Some "pocket change" of frequency, a few $\mathrm{THz}$, could be synthesized as sidebands using a Kourogi comb, based on a microwave modulator in a cavity whose length provided resonance enhancement of all the generated sidebands (Kourogi et al., 1996). In such a way we measured the $532 \mathrm{~nm}$ Iodine standard in terms of the difference of frequency between twice the HeNe Iodine system at $633 \mathrm{~nm}$, and the Rb two-photon line at 782 nm (Hall et al., 1999).

This was our introduction to the elegance of having an optical comb-a coherent ensemble of spectral lines whose frequencies are accurately represented by a simple formula. Our system covered just a few nm wavelength. How sweet it would be to cover the entire visible band, giving several million accurately known frequency reference lines all at once.

One way to broaden this Kourogi comb's spectral width would be to provide intracavity gain, to compensate the modulator's optical losses, a scheme which was demonstrated by Diddams using an OPO crystal also inside the resonator. Oscillation and generation of hundreds of frequency modulation (FM) sidebands were easily observed (Diddams et al., 1999). For some tuning conditions the phase of the several spectral components led to pulse generation, rather than pure FM emission. In many ways this was just the hard way to do what the UltraFast Laser scientists appreciated about the Ti:Sapphire self-mode-locked lasers: stable, selforganized, ultrashort high repetition rate pulse trains. Elsewhere our group's papers discuss the technical richness of these lasers and the comb business (Hall et al., 2001). This is just one further note about the mutual coupling between "independent" research streams: we switched to Ti:Sapphire fs lasers and never looked back.

Coincidentally, in these final days of the last Millennium, this laser community received a fundamentallyimportant gift from the laser industry. There would probably be no widely-used frequency combs without it. This "gift" was the introduction of high-power visible lasers, based on frequency-doubling the output of a laser-diode-pumped Nd solid-state laser. These were immediately put to use replacing the fussy and quite noisy Argon Ion laser in wide use for pumping the Solid-State lasers. Competitive forces led these new pump lasers to be well engineered, with intensity stabilization to yield exceedingly low levels of residual amplitude noise. This property is crucial because of the way a self-modelocked laser operates - these Ti:Sapphire lasers are selfmode-locked by a self-induced optical lens which makes the cavity less lossy when the laser modes are all synchronized to form an "optical bullet" in the laser medium (Salin et al., 1991). This temporary lens is formed by the radial index gradient, induced and present only if a light bullet is present. So the laser cavity is originally set up to need this extra focusing to produce low-loss cavity modes, and after the laser is started in the pulse regime, stable self-mode-locking is maintained. Consider that the pulse lengths are only $\sim 10 \mathrm{fs}$, while the repetition periods are $\sim 10 \mathrm{~ns}$. With ideal synchronization, the peak power/average power ratio is $\sim 10^{6}$. A typical laser will emit $\sim 0.5 \mathrm{~W}$ through an output mirror of $5 \%$ transmission. So we have $10 \mathrm{~W}$ average internal power, and $10 \mathrm{MW}$ peak power, which is focused to a $\sim 14 \mu \mathrm{m}$ radius spot in the Ti:Sapphire laser crystal. This active area is only $3 \times 10^{-6} \mathrm{~cm}^{2}$, so with $10 \mathrm{MW}$ peak power we have $3 \mathrm{TW} / \mathrm{cm}^{2}$. The associated electric field is $\sim 10 \%$ of the interatomic fields in the crystal, so it is not so surprising that a significant optically-induced increase of the index of refraction occurs (optical Kerr effect). The low amplitude noise of the pump laser is now seen to be critical: an intensity-dependent phase-shift though the laser crystal will produce amplitude $\rightarrow$ frequency conversion and thus unacceptable phase noise if the pump is noisy. In a good case the linewidth of laser comb-lines without frequency control is $\sim 3-10 \mathrm{kHz}$ due to this cause, before the servo is used. Details of the process have been studied (Holman et al., 2003).

So the pulse train leaving the laser is of $\sim 500 \mathrm{~kW}$ peak power, much of which we will focus into the special nonlinear fibers that brought in the age of the Optical Comb. Because of the microstructure design of the fiber, full light guiding is possible even with fiber core sizes of $1.5-2 \mu \mathrm{m}$ diameter. So now when we estimate the fiber's active area, it is roughly 200 -fold smaller than the laser's, while the power level is $\sim 20$-fold lower. The tenfold higher intensity produces a threefold higher electric field in the silica fiber, being now essentially comparable with interatomic field and setting the stage for serious NonLinear interactions. Forget Taylor's expansion here: this is strong signal NonLinear physics. All frequency components from the laser are mixed with each other, resulting in a drastic spectral broadening. By the fiber's optical design, a broad range of optical frequencies can travel through the fiber with little speed variation, which allows these frequency conversion processes to remain phase-matched and accumulate power into the newly created frequencies. Essentially, in a few $\mathrm{cm}$ of length, the input spectrum is converted to white light and covers 
an octave or more of optical bandwidth. Actually the light is not quite "white" since it still carries the basic heartbeat of the original fs laser, for example, $100 \mathrm{MHz}$. As explained previously, this intrinsically generates a comb spectrum with component widths just connected to the spectral resolving power employed. Eventually, at the $\mathrm{kHz}$ level and below, the broadly-active phase modulation processes that affect all lasers will broaden these lines also (before the servo-control is $\mathrm{ON}$ ).

\section{COMPLEMENTARITY, COOPERATION, AND COMPETITION}

The basics

The remarkable insights of Hänsch's Stanford work (Eckstein et al., 1978) were published in 1978, and already demonstrated using a repetitively-pulsing laser to generate an optical comb which could serve as a spectral ruler. However, the bandwidth of the covered spectrum was too small for general frequency measurementsonly a $\mathrm{GHz}$ or two. Since these intervals could be spanned in other ways, the methods were not widely adopted. Basically there was not a technical growth path available at the time. Principle, yes; Tool, no.

The hard work, straight-ahead "government" approach to frequency measurement had been demonstrated at NBS in 1972 (Evenson et al., 1972), following the pioneering work of Ali Javan's MIT frequency measurement group [see references in Sanchez et al. (1978)]. But this was a heroic effort and mainly only national standards laboratories took much interest. Laser after different laser had to be lined up and frequency-related to the doubled frequency of its predecessor, to step-bystep build up the frequency measurement chain. This kind of work required development of frequency- and phase-locking schemes now in wide use. We also got a "one-of-a-kind" physical result, a single laser frequency was measured by the cooperative and extended work of the NBS group (Jennings et al., 1983). But it was enough to get the Metre redefinition process started.

\section{The divide and conquer scheme}

In a notable paper (1990), Hänsch and his colleagues suggested an excellent way to simplify the frequency chains: one should use the difference frequencies between lasers as the entities that were harmonically marching up the spectrum (Telle et al., 1990). In this way, the ensemble of lasers would all have nearly the same wavelength, and could be built essentially by duplication of a basic diode laser unit. Then with nonlinear crystals, fast photodetectors, and suitable phase-locking electronics one could progress from microwaves to optical frequencies. This system also felt rather elaborate and specialized, but was used with good results in Garching. A related strategy was developed at NRC (Whitford, 1980), based on difference frequencies, using $\mathrm{CO}_{2}$ lasers. Inspecting such a system, one came to see that the first 9 or 10 of the 14 stages served only to get the frequency up into the low $\mathrm{THz}$ range.

Then in 1994 came Kourogi and Ohtsu's multiplyresonant cavity approach, allowing one to reach a few $\mathrm{THz}$ in a single step (Kourogi et al., 1996). Eventually the buildup of phase noise-according to the high harmonic of the original microwave source-would have been a problem in going into the visible range. But the fs laser Comb arrived and offers an easier and better way. See below.

\section{A brief history of the optical miracle of 1999-2000}

\section{Fibers for spectral broadening}

By now the JILA group had accepted the fs laser as a great source of pulsed laser light. Ours had $\sim 80 \mathrm{~nm}$ bandwidth at $800 \mathrm{~nm}$. But the optical frequency standards we wanted to connect were at $1064 \mathrm{~nm}$ (fundamental of Iodine-stabilized $\mathrm{Nd}$ laser) and $778 \mathrm{~nm}(\mathrm{Rb}$ two-photon-stabilized diode laser). An ordinary communication fiber was found to be just barely capable of spectral broadening the necessary amount-104 THz. This paper was submitted at the end of September 1999 (Diddams et al., 2000).

\section{Microstructure fibers for serious nonlinearity}

The Conference on Laser and Electro-Optics of June 1999 had a spectacular postdeadline presentation by a Bell Labs team (Ranka and Stentz, 2000), wherein a normal fs laser pulse evolved its color in a dramatic way in propagating through a few meters of a special fiber. Such a fiber did make collimated white light, in the form of stably-repeating pulses, just as Ted Hänsch had postulated for his (unpublished) frequency measurement proposal. Using that previously-unknown light source, most of the rest should be possible. (Seeing the repetitivelypulsed laserlike white light the fiber generated instantly convinced me that Hänsch's Concept actually could be a real and physical possibility. Without a repetitive whitelight laser, there was no chance.) Lengthy appeals for scientific collaboration with the fiber owners' organization ultimately became irrelevant due to the miraculous appearance in JILA of a sample of this Magic Fiber. The concept of "band-gap" or "Photonic-Crystal" fibers was introduced in 1996 by Knight et al., pointing out the possibility of controlling the spatial modes and effective group velocity dispersion by the mechanical design of the air holes (Knight et al., 1996). Our first JILA experiments were made using microstructured fiber drawn from a preform prepared on 10 September, 1997 by Robert S. Windeler of Bell Labs (Windeler, 2006), using a construction technique of his own devising. A broad range of fiber designs was investigated in Bath, UK, by P. St. J. Russell and colleagues.

\section{The race is $\mathrm{ON}$}

Of course in JILA we did not know that the Garching team had already gone from a plan to the first demon- 
TABLE I. Measured optical frequencies: The reference atom or molecule and its transition wavelength are indicated, followed by lead author and institution, the journal name, and date. The first fs Comb measurement was Hydrogen by Reichert et al. The first direct fs optical measurements were by the JILA team (Jones). Note the brevity of time between publications.

\begin{tabular}{ccclcc}
\hline \hline $\mathrm{Ca}$ & $657 \mathrm{~nm}$ & Schnatz & PTB & PRL & 1 Jan '96 \\
$\mathrm{Rb}$ & $780 \mathrm{~nm}$ & Ye & JILA & Opt. Lett. & August '96 \\
$\mathrm{C}_{2} \mathrm{H}_{2}$ & $1500 \mathrm{~nm}$ & Nakagawa & NRLM & JOSA-B & Dec '96 \\
$\mathrm{I}_{2}$ & $532 \mathrm{~nm}$ & Hall & JILA & IEEE Instrum. Meas. & April '99 \\
$\mathrm{Sr}^{+}$ & $674 \mathrm{~nm}$ & Bernard & NRC & PRL & 19 Apr '99 \\
$\mathrm{In}^{+}$ & $236 \mathrm{~nm}$ & v. Zanthier & MPQ & Opt. Commun. & Aug '99 \\
$\mathrm{H}$ & $243 \mathrm{~nm}$ & Reichert & MPQ & PRL & 10 Apr '00 \\
$\mathrm{Rb}$ & $778 \mathrm{~nm}$ & D. Jones & JILA & Science & 28 Apr '00 \\
$\mathrm{I}_{2}$ & $532 \mathrm{~nm}$ & Diddams & JILA & PRL & 29 May '00 \\
$\mathrm{H}$ & $243 \mathrm{~nm}$ & Niering & MPQ & PRL & 12 June '00 \\
$\mathrm{Yb}$ & $467 \mathrm{~nm}$ & Roberts & NPL & PRA & 7 July '00 \\
$\mathrm{In}^{+}$ & $236 \mathrm{~nm}$ & v. Zanthier & MPQ & Opt. Lett. & 1 Dec '00 \\
$\mathrm{Ca}^{+}$ & $657 \mathrm{~nm}$ & Stenger & PTB & PRA & 17 Jan '01 \\
$\mathrm{Hg}^{+}$ & $282 \mathrm{~nm}$ & Udem & NIST & PRL & 28 May '01 \\
$\mathrm{Ca}^{6}$ & $657 \mathrm{~nm}$ & Udem & NIST & PRL & 28 May '01 \\
$\mathrm{Yb}^{+}$ & $435 \mathrm{~nm}$ & Stenger & PTB & Opt. Lett. & 5 Oct '01 \\
\hline \hline
\end{tabular}

stration of a comb-based phase coherent link from microwaves to the visible, and had submitted their Phys. Rev. Lett. in November 1999. Even before we got the Magic Fiber. They used a comb of somewhat limited bandwidth, $44 \mathrm{THz}$, but their divider stages could connect the optical frequency with the 28th harmonic of the difference between the comb's edges. It is a beautiful result, and appeared finally on 10 April 2000 (Reichert et al., 2000). In the meantime the JILA team was working hard with the Magic Fiber's white-light output to implement and demonstrate our phase-coherent locking of the carrier-envelope offset frequency in terms of the laser's repetition rate. Our Disclosure of the scheme called this "Self-Referencing." The control electronics we built had a digital click switch so the phase could be set on any integer multiple of $1 / 16$ of a cycle of phase-slip per pulse. The JILA experimental demonstration was based on interferometrically determining the carrier-envelope phase difference between two optical pulses, separated by one intervening pulse. Finally the new electronics worked, the experimental data were clear, and our report (Jones et al., 2000) appeared in Science on 28 April 2000. A Phys. Rev. Lett. joint article celebrated the success of the combined Garching, Bell Labs, and JILA teams and appeared on 29 May 2000 (Diddams et al., 2000). Within the next year there was an avalanche of absolute optical frequency measurements from labs all over the world. This was a glorious chapter in optical physics history, in no small part because of the high mutual respect of the two teams for each other, aided by the complete openness fostered by the frequent exchange of postdocs Scott Diddams and Thomas Udem between the two hotly-competing groups.

\section{Some frequency measurement results}

Many laser frequency standards were being actively studied worldwide so that, when the Comb breakthrough came, there were many things to be accurately measured-many for the first time. A few of the worldwide results include the work shown in Table I.

The comb technology spread explosively in 2000, bringing vast simplification of optical frequency measurements, along with a steady improvement in the accuracy. Very soon after the initial measurements, it has become the case that the comb's measurement precision can exceed that of the standards being measured. Recent tests at NIST, BIPM, and ECNU (Ma et al., 2004) confirm the earlier MPQ experiments (Udem et al., 2002) showing that the comb principle is strictly correct up to a measurement precision of more than 18 digits.

\section{Molecular iodine optical frequency standard}

The Iodine-stabilized Nd:YAG laser is a sweet spot in the stabilized laser domain, based on its excellent performance and relative simplicity. One system was made in Japan that met airlines' cabin baggage limitations and still delivered excellent performance (Hong et al., 2003). Because of Iodine's great atomic mass, the second-order Doppler correction for this system is only $\sim 5 \times 10^{-13}$ and it is likely that independent reproducibility perhaps fivefold superior to this can come with improved technical realizations. In particular, providing an offset-free modulation strategy is still a challenge. The advantage of this system is its compactness and potentially reasonable cost. Taken with an optical comb, one can have an attractive clock (Ye et al., 2001) (see Fig. 4). The frequency (in)stability of all the $1 \times 10^{6}$ optical comb lines is $\sim 4$ $\times 10^{-14} / \sqrt{\tau}$. 


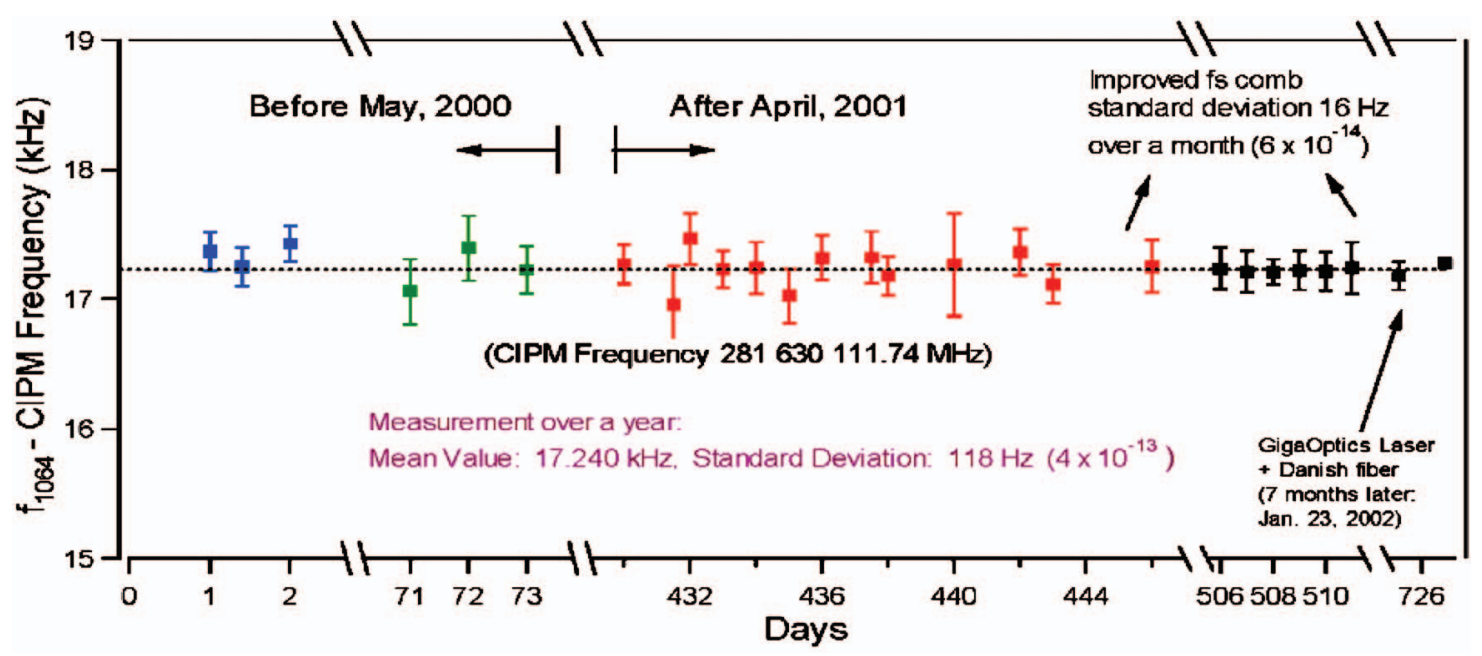

FIG. 4. (Color) Long-term frequency stability of Iodine-based Optical Clock. This conveys the refinement and small frequency offset of this stable optical clock's frequency from previous, much less accurate measurements. With improved technology in 2002 the uncertainty was further reduced to $\sim 6 \times 10^{-14}$.

Recently stable Yb:YAG single frequency lasers became available, with output tunable to $1029 \mathrm{~nm}$. When frequency doubled, excellent stabilization performance should be possible with the $\mathrm{I}_{2}$ transitions at $514.5 \mathrm{~nm}$, considering that the linewidth is at least fivefold smaller than for the $532 \mathrm{~nm}$ line (Cheng et al., 2002). Single frequency fiber systems can also offer this wavelength.

\section{SO WHAT COMES NEXT?}

In addition to the simplification of optical frequency measurements, the resulting new capabilities are unbelievably rich in terms of the tools and capabilities that have been created, and these in turn are reinforcing progress in these contributing fields. This paper cannot even attempt to present a myriad of delicious physical effects, which are normally understood as being in different fields, but which in their now-unified relationships can be seen as creating a truly remarkable and enabling advance of the research tools available in optical science. But let me still give a few examples.

After the frenzy of Generation I frequency measurements of Table I, some of the Generation II comb applications in Jun Ye's group include low-jitter time synchronization $(\sim \mathrm{fs})$ between ultrafast laser sources (Shelton et al., 2002), coherent stitching-together the spectrum of separate fs laser sources so as to spectrally broaden and temporally shorten the composite pulse (Shelton et al., 2001), precision measurement of optical nonlinearities using the phase measurement sensitivity of rf techniques (Fortier et al., 2003), coherently storing a few hundred sequential pulses and then extracting their combined energy to generate correspondingly more intense pulses at a lower repetition rate (Jones and Ye, 2004), and searching for a change in the physical constants by the Garching team (Fischer et al., 2004). Exciting topics of research for Generation III applications now include connecting optical frequency interim standards at the sub-Hz level (in spite of their different locations spectrally and physi- cally), allowing precise remote synchronization of accelerator cavity fields, providing stable reference oscillators for Large Array Microwave Telescopes, and potentially reducing the relative phase noise of the oscillator references used for deep space telescope arrays (NASA, VLBI, etc.) That's part of the first five years.

And the next projects? What about $14.4 \mathrm{keV}$ combline harmonics to look at Mössbauer ${ }^{57} \mathrm{Fe}$ nuclear resonances? Another sharp line is in ${ }^{181} \mathrm{Ta}$ at $6.2 \mathrm{keV}$. How about parallel processing to determine biological activity of a candidate drug, by means of coherent anti-Stokes Raman spectroscopy using synchronized pulse lasers to excite specific ligand Raman resonances of a single molecule that was attracted to and stuck by a particular test protein patch on a surface?

In a larger framework, we now find ourselves at an almost unique point in the development of Science, where we have the remarkable ability to "understand" practically all phenomena, to compute accurate predictions from our equations, and to integrate a variety of details into our models. Consider, for example, the GPS system, in which different kinds of physics such as gravity and relativity are successfully merged with our sophisticated atomic clocks - not to forget satellite dynamics, radio engineering, and computer software-so that in the total we have a coherent and highly useful practical tool. Remarkably, the system is simple for the end user to apply. We must count this GPS achievement as one of the all-time ultimate technical success levels ever achieved.

The work recognized by the 2005 Nobel Physics Prize represents entry of another dramatic, major, and enabling advance, and one which we can expect to show some flavors of the same breadth and character just noted regarding GPS. But in these first moments after its birth, our optoelectronic technology is new and is barely illustrated, not much beyond the first cases of interest to frequency-standard people and metrologists. We know that the accuracy of optical frequency mea- 
surements is now limited to "just" 15 digits by the present microwave standard of frequency, but the Comb technology actually allows two optical frequencies to be compared with several orders of magnitude more precision. If the history of physics is any guide, we realistically can expect to find some nice surprises ahead as these capabilities become even more widespread, and are applied to ingenious fundamental measurements by a growing and imaginative community of "fundamental physics" scientists. After considering all the known progress in Science, would you bet that we have already opened the Russian Matryoshka doll of Nature and already found the ultimate inside limit?

\section{ACKNOWLEDGMENTS}

The joy of interacting with superb young scientists is clearly one of life's treasures. Among these many I must single out for special thanks Jim Bergquist, Leo Hollberg, Miao Zhu, and Jun Ye for their enthusiasm and unique contributions to the JILA program. The NIST management is enthusiastically thanked for accepting and sponsoring over the years a series of risky proposals in Laser Spectroscopy. Particularly Leo Hollberg, Steve Cundiff, and I were glad they accepted the fs comb frequency synthesizer proposal in Spring 1999. Scott Diddams and David Jones were the excellent colleagues additionally involved in these experiments, and JILA's research force was hugely expanded when Jun Ye rejoined JILA in 1999 to start his own group. As always, whenever Professor Long Sheng Ma was visiting us from Shanghai our pace was strongly advanced. Visiting Scientists such as Christian Bordé continue to be collaborators even three decades after their JILA time. I am pleased and grateful to acknowledge that the work has been supported in part by the NSF, ONR, AFOSR, and NASA, and for over four decades by the NIST. I benefited greatly from the knowledge and generous sharing of ideas and opportunities by my NBS mentor, Peter L. Bender. Above all I am indebted to my patient and insightful lifetime friend Lindy Hall for her understanding, her great efforts and contributions to this scientific work, and, more importantly, to our joyous and fun life together. It has been wonderful in the course of these 45 years to see a progression of experiments and technical advances make possible this ultimate payoff in the optical comb.

\section{APPENDIX: THE FULL COMB STORY IN AN UNDERGRADUATE'S WORLD}

I'm glad you asked how to think about frequency combs. Suppose you have a sine-wave voltage or field. Then a plot in time shows a smooth oscillation and a plot in frequency shows a single Fourier component, namely, a sharp line. Now add a few harmonics onto this wave. The spectrum now has a few more lines at exact harmonic frequencies, while the time picture has a rather complicated shape. By adjusting the phases of the harmonics, we can begin to synthesize some disturbance in time that begins to remind one of a pulse, or, more exactly, a series of identical pulses. Carry this a step forward by having a large number of harmonics. The more we add, the sharper is the pulse we can synthesize, and of course the richer is the spectrum of this wave. Going further in this direction of adding coherent harmonics, the spectrum now has zillions of spectral lines, all at the harmonics of our original sinewave. Carrying this concept to the visible will require a few million $\left(10^{6}\right)$ harmonics for a source with $100 \mathrm{MHz}$ basic repetition rate. With the proper phase adjustments, the time domain pulse can be $10^{6}$ times sharper in time than the original sine wave. So we can expect really narrow temporal pulses, and really wide spectral bandwidths.

This situation fits well with what we would expect from Fourier analysis of a single pulse: such an impulse will have Fourier components at all frequencies, with their nearly-constant amplitudes gradually decreasing for frequencies above the reciprocal temporal pulse width. If we have a repetitive pulse train in time, but insist to ask about its spectrum, we will need an analyzer with a narrower passband compared with the repetition frequency, otherwise it could not resolve the harmonic structure. But a narrow spectral passband corresponds to a long temporal response time. So the output of the spectrometer at any particular wavelength or frequency setting will be the result of coherent addition of the contributions of many pulses. While an individual pulse has a broad and continuous spectrum, when we coherently add their spectral amplitudes we can expect to have interferences that will modulate the spectrum. Adding more pulses temporally (narrower spectral resolution) will give deeper modulation. Eventually we arrive at very sharp spectral lines, evenly arranged as Fourier harmonics. Until we encounter technical issues such as phase-noise of the repetition rate, the sharper an analysis resolution we apply to the waveform, the sharper will be the spectral lines we observe. So the spectrum does indeed remind one of a "comb." You can demonstrate these ideas safely at home for yourself easily in the electronics domain, but of course the optical and electronics worlds should work the same.

In fact, with the fs lasers used to generate these pulses, there is one more little item of interest. That is that the laser can oscillate in any one of its cavity modes, defined by having a repeating phase after going one loop around the cavity. All the many modes involved have their own longitudinal quantum numbers, essentially how many full optical cycles are contained in the closed loop. This calculation clearly involves the wavelengthdependent phase velocity, and some average of the propagation through the many optical components. Another reality is that the laser operates in a self-organized repetitive pulsing mode. Effectively the laser's optical losses can be made large enough to inhibit laser action, unless all the cavity modes can adjust their phases to synthesize a delta-function spatially. The critical thing is to have a short pulse when passing through the Ti:Sapphire crystal, since the short pulse will correspond to very high peak power, and that will interact with the laser rod's material in a quadratic way (optical Kerr ef- 
fect) to produce a positive lens: a bigger index on the axis where the intensity is maximum. So the selforganized pulse situation is stable in which the laser's cavity has a high diffraction-loss (does not have quite enough positive lens power), but the losses are periodically remedied by a bullet of light which uses its selfaction on the crystal to produce the needed extra refraction that makes the cavity losses be suitably less.

Now the pulse envelope that describes this light "bullet" results from superposition of many cavity modes, and the shape will evolve if there are temporal delay differences with wavelength. We are now just discussing the group velocity concept, whereby the shape of a disturbance will evolve unless all the frequencies have the same propagation speed. In the physical laser we must include some optical elements specifically to deal with the fact that blue light in the laser crystal will travel more slowly than red light. To get the shortest pulses the time delays around the loop need to be essentially the same, although you can see this becomes a little complicated in that the laser pulses themselves act to influence the time delays. In any case, the light which comes out of the laser's coupling mirror will be a regular time series of sharp pulses, and will display a comb-like structure under frequency analysis. However, the underlying fast optical oscillations will in general have a different phase each time the pulse hits the mirror's surface. The fast oscillation's phase will shift a bit forward or backward from one pulse to the next, and so the optical frequency comb may be offset a bit from the strictly Fourier harmonic case we first imagined. The usual case is a constant phase shift for each pulse, and so a constant rate of accumulating a phase beyond the repetition-rate's harmonic. We have developed an electro-optic scheme called "self-referencing" in which this additional frequency, the Carrier-Envelope Offset Frequency, is stably locked to the repetition frequency in a digital ratio. For example, one could choose zero for the set-point ratio and thereby have a strictly harmonic comb. With the offset $=1 / 2$, one generates a comb offset by $1 / 2$ the basic repetition rate, which itself is of course the frequency comb's tooth spacing. ${ }^{1}$

\section{REFERENCES}

Bagaev, S. N., L. S. Vasilenko, A. K. Dmitriev, M. N. Skvortsov, and V. P. Chebotaev, 1976, "Narrowing of nonlinear resonances in low pressure gases," JETP Lett. 23, 360363.

Bagayev, S. N., V. P. Chebotayev, A. K. Dmitriyev, A. E. Om, Y. V. Nekrasov, and B. N. Skvortsov, 1991, "2nd-order Doppler-free spectroscopy," Appl. Phys. B: Photophys. Laser Chem. 52, 63-66.

Barger, R. L., and J. L. Hall, 1969, "Pressure shift and broadening of methane line at 3.39 um studied by laser-saturated molecular absorption," Phys. Rev. Lett. 22, 4-8.

Bethlem, H. L., G. Berden, and G. Meijer, 1999, "Decelerating

\footnotetext{
${ }^{1}$ See Diddams et al., 2000; Jones et al., 2000; Reichert et al., 2000; Udem et al., 2002; Ma et al., 2004.
}

neutral dipolar molecules," Phys. Rev. Lett. 83, 1558-1561. BIPM, http://www.BIPM.org/en/si/si_brochure/

Borde, C. J., 2005, "Base units of the SI, fundamental constants and modern quantum physics," Philos. Trans. R. Soc. London, Ser. A 363, 2177-2201.

Borde, C. J., J. L. Hall, C. V. Kunasz, and D. G. Hummer, 1976, "Saturated absorption line shape: Calculation of the transit-time broadening by a perturbation approach," Phys. Rev. A 14, 236-263.

Cheng, W. Y., L. S. Chen, T. H. Yoon, J. L. Hall, and J. Ye, 2002, "Sub-Doppler molecular-iodine transitions near the dissociation limit (523-498 nm)," Opt. Lett. 27, 571-573.

Clairon, A., P. Laurent, G. Santarelli, S. Ghezali, S. N. Lea, and M. Bahoura, 1995, "A cesium fountain frequency standard-Preliminary results," IEEE Trans. Instrum. Meas. 44, 128-131.

Diddams, S. A., D. J. Jones, L. S. Ma, S. T. Cundiff, and J. L. Hall, 2000, "Optical frequency measurement across a 104$\mathrm{THz}$ gap with a femtosecond laser frequency comb," Opt. Lett. 25, 186-188.

Diddams, S. A., D. J. Jones, J. Ye, T. Cundiff, J. L. Hall, J. K. Ranka, R. S. Windeler, R. Holzwarth, T. Udem, and T. W. Hansch, 2000, "Direct link between microwave and optical frequencies with a $300 \mathrm{THz}$ femtosecond laser comb," Phys. Rev. Lett. 84, 5102-5105.

Diddams, S. A., L.-S. Ma, J. Ye, and J. L. Hall, 1999, "Broadband optical frequency comb generation with a phasemodulated parametric oscillator," Opt. Lett. 24, 1747-1749.

Drever, R. W. P., J. L. Hall, F. V. Kowalski, J. Hough, G. M. Ford, A. J. Munley, and H. Ward, 1983, "Laser phase and frequency stabilization using an optical-resonator," Appl. Phys. B: Photophys. Laser Chem. 31, 97-105.

Eckstein, J. N., A. I. Ferguson, and T. W. Hansch, 1978, "Highresolution 2-photon spectroscopy with picosecond lightpulses," Phys. Rev. Lett. 40, 847-850.

Evenson, K. M., J. S. Wells, F. R. Petersen, B. L. Danielson, G. W. Day, R. L. Barger, and J. L. Hall, 1972, "Speed of light from direct frequency and wavelength measurements of the methane-stabilized laser," Phys. Rev. Lett. 29, 1346-1349.

Fischer, M., N. Kolachevsky, M. Zimmermann, R. Holzwarth, T. Udem, T. W. Hansch, M. Abgrall, J. Grunert, I. Maksimovic, S. Bize, H. Marion, F. P. Dos Santos, P. Lemonde, G. Santarelli, P. Laurent, A. Clairon, C. Salomon, M. Haas, U. D. Jentschura, and C. H. Keitel, 2004, "New limits on the drift of fundamental constants from laboratory measurements," Phys. Rev. Lett. 92, 230802.

Flowers, J. L., and B. W. Petley, 2001, "Progress in our knowledge of the fundamental constants of physics," Rep. Prog. Phys. 64, 1191-1246.

Fortier, T. M., D. J. Jones, J. Ye, and S. T. Cundiff, 2003, "Highly phase stable mode-locked lasers," IEEE J. Sel. Top. Quantum Electron. 9, 1002-1010.

Gibble, K., and S. Chu, 1993, "Laser-cooled Cs frequency standard and a measurement of the frequency-shift due to ultracold collisions," Phys. Rev. Lett. 70, 1771-1774.

Hall, J. L., 1968, "The laser absolute wavelength standard problem,” IEEE J. Quantum Electron. QE-4, 638-641.

Hall, J. L., 2000, “Optical frequency measurement: 40 years of technology revolutions," IEEE J. Sel. Top. Quantum Electron. 6, 1136-1144.

Hall, J. L., C. J. Bordé, and K. Uehara, 1976, "Direct optical resolution of recoil effect using saturated absorption spectroscopy," Phys. Rev. Lett. 37, 1339-1342. 
Hall, J. L., L.-S. Ma, M. Taubman, B. Tiemann, F.-L. Hong, O. Pfister, and J. Ye, 1999, "Stabilization and frequency measurement of the $I_{2}$-stabilized Nd:YAG laser," IEEE Trans. Instrum. Meas. 48, 583-653.

Hall, J. L., J. Ye, S. A. Diddams, L.-S. Ma, S. T. Cundiff, and D. J. Jones, 2001, "Ultrasensitive spectroscopy, the ultrastable lasers, the ultrafast lasers, and the seriously nonlinear fiber: A new alliance for physics and metrology," IEEE J. Quantum Electron. 37, 1482-1492.

Heavner, T. P., S. R. Jefferts, E. A. Donley, J. H. Shirley, and T. E. Parker, 2005, "NIST-F1: recent improvements and accuracy evaluations," Metrologia 42, 411-422.

Holman, K. W., R. J. Jones, A. Marian, S. T. Cundiff, and J. Ye, 2003, "Detailed studies and control of intensity-related dynamics of femtosecond frequency combs from mode-locked Ti:sapphire lasers," IEEE J. Sel. Top. Quantum Electron. 9, 1018-1024.

Hong, F. L., J. Ishikawa, K. Sugiyama, A. Onae, H. Matsumoto, J. Ye, and J. L. Hall, 2003, "Comparison of independent optical frequency measurements using a portable iodinestabilized Nd:YAG laser," IEEE Trans. Instrum. Meas. 52, 240-244.

Hudson, E. R., H. J. Lewandowski, B. C. Sawyer, and J. Ye, 2006, "Cold molecule spectroscopy for constraining the evolution of the fine structure constant," Phys. Rev. Lett. 96, 143004.

Jennings, D. A., C. R. Pollock, F. R. Petersen, R. E. Drullinger, K. M. Evenson, J. S. Wells, J. L. Hall, and H. P. Layer, 1983, "Direct frequency-measurement of the $I_{2}$-stabilized He-Ne 473-THz (633-nm) laser," Opt. Lett. 8, 136-138.

Jones, D. J., S. A. Diddams, J. K. Ranka, A. Stentz, R. S. Windeler, J. L. Hall, and S. T. Cundiff, 2002, "Carrierenvelope phase control of femtosecond mode-locked lasers and direct optical frequency synthesis," Science 288, 635-639.

Jones, R. J., and J. Ye, 2004, "High-repetition-rate coherent femtosecond pulse amplification with an external passive optical cavity," Opt. Lett. 29, 2812-2814.

Kasevich, M. A., E. Riis, S. Chu, and R. G. Devoe, 1989, "Rf spectroscopy in an atomic fountain," Phys. Rev. Lett. 63, 612616.

Knight, J. C., T. A. Birks, P. S. Russell, and D. M. Atkin, 1996, "All-silica single-mode optical fiber with photonic crystal cladding," Opt. Lett. 21, 1547-1549.

Kourogi, M., T. Enami, and M. Ohtsu, 1996, “A coupled-cavity monolithic optical frequency comb generator," IEEE Photonics Technol. Lett. 8, 1698-1700.

Letokhov, V. S., and V. P. Chebotayev, 1977, NonLinear Laser Spectroscopy (Springer-Verlag, Berlin).

Levenson, M. D., and S. S. Kano, 1988, Introduction to NonLinear Laser Spectroscopy (Academic, San Diego).

Ma, L. S., Z. Y. Bi, A. Bartels, L. Robertsson, M. Zucco, R. S. Windeler, G. Wilpers, C. Oates, L. Hollberg, and S. A. Diddams, 2004, "Optical frequency synthesis and comparison with uncertainty at the 10(-19) level," Science, 303, 1843 1845.

Ma, L. S., J. Ye, P. Dubé, and J. L. Hall, 1999, "Ultrasensitive frequency-modulation spectroscopy enhanced by a highfinesse optical cavity: Theory and application to overtone transitions of $\mathrm{C} 2 \mathrm{H} 2$ and C2HD," J. Opt. Soc. Am. B 16, 2255-2268.

NIST, http://www.mel.nist.gov/div821/museum/timeline.htm

Notcutt, M., L. S. Ma, J. Ye, and J. L. Hall, 2005, "Simple and compact $1-\mathrm{Hz}$ laser system via an improved mounting con- figuration of a reference cavity," Opt. Lett. 30, 1815-1817.

Polzik, E. S., J. Carri, and H. J. Kimble, 1992, “Atomic spectroscopy with squeezed light for sensitivity beyond the vacuum-state limit," Appl. Phys. B: Photophys. Laser Chem. 55, 279-290.

Ranka, R. W. J., and A. Stentz, 2000, "Visible continuum generation in air-silica microstructure optical fibers with anomalous dispersion at $800 \mathrm{~nm}$," Opt. Lett. 25, 25.

Reichert, J., M. Niering, R. Holzwarth, M. Weitz, T. Udem, and T. W. Hansch, 2000, "Phase coherent vacuum-ultraviolet to radio frequency comparison with a mode-locked laser," Phys. Rev. Lett. 84, 3232-3235.

Salin, F., J. Squier, and M. Piche, 1991, "Mode locking of Ti: Sapphire lasers and self-focusing: A Gaussian approximation," Opt. Lett. 16, 1674-1478.

Sanchez, A., C. F. Davis, K. C. Liu, and A. Javan, 1978, "Mom tunneling diode-Theoretical estimate of its performance at microwave and infrared frequencies," J. Appl. Phys. 49, 5270-5278.

Shelton, R. K., S. M. Foreman, L. S. Ma, J. L. Hall, H. C. Kapteyn, M. M. Murnane, M. Notcutt, and J. Ye, 2002, "Subfemtosecond timing jitter between two independent, actively synchronized, mode-locked lasers," Opt. Lett. 27, 312-314.

Shelton, R. K., L.-S. Ma, H. C. Kapteyn, M. M. Murnane, J. L. Hall, and J. Ye, 2001, "Phase-coherent optical pulse synthesis from separate femtosecond lasers," Science 293, 1286-1289.

Sobel, D., 1995, Longitude, the True Story of a Lone Genius Who Solved the Greatest Scientific Problem of His Time (Penguin Books, New York).

Steiner, R., E. R. Williams, D. B. Newell, and R. Liu, 2005, "Towards an electronic kilogram: An improved measurement of the Planck constant and electron mass," Metrologia 42, 431-441.

Stenholm, S., 1984, Foundations of Laser Spectroscopy (Wiley, New York).

Svanberg, S., 1992, Atomic and Molecular Spectroscopy, 2nd ed. (Springer-Verlag, Berlin).

Telle, H. R., D. Meschede, and T. W. Hansch, 1990, "Realization of a new concept for visible frequency-division-Phase locking of harmonic and sum frequencies," Opt. Lett. 15, 532-534.

Udem, T., R. Holzwarth, and T. W. Hansch, 2002, "Optical frequency metrology," Nature (London) 416, 233-237.

Whitford, B. G., 1980, "Measurement of the absolute frequencies of CO2-laser transitions by multiplication of $\mathrm{CO} 2$-laser difference frequencies," IEEE Trans. Instrum. Meas. 29, 168176.

Windeler, R. S., 2006, private communication with J. L. Hall. Ye, J., L. S. Ma, and J. L. Hall, 2001, "Molecular iodine clock," Phys. Rev. Lett. 87, 270801.

Young, B. C., F. C. Cruz, W. M. Itano, and J. C. Bergquist, 1999, "Visible lasers with subhertz linewidths," Phys. Rev. Lett. 82, 3799-3802.

Young, B. C., R. J. Rafac, J. A. Beall, F. C. Cruz, W. M. Itano, D. J. Wineland, and J. C. Bergquist, 2005, "Hg+ optical frequency standard: Recent progress," in ICOLS 2005, Vol. XVII, Laser Spectroscopy (World Scientific, Cairngorm, Aviemore, Scotland), p. 433.

Ziskind, M., C. Daussy, T. Marrel, and C. Chardonnet, 2002, "Improved sensitivity in the search for a parity-violating energy difference in the vibrational spectrum of the enantiomers of CHFCIBr," Eur. Phys. J. D 20, 219-225. 